\title{
SUPERCONDUCTING INTERMEDIATE-VELOCITY CAVITY DEVELOPMENT FOR RIA
}

\author{
K.W. Shepard, M.P. Kelly, J.D. Fuerst, M. Kedzie, ANL, Argonne IL 60439, USA
}

\begin{abstract}
This paper discusses the design and development of two types of intermediate-velocity superconducting cavity and an associated cryomodule for the RIA driver linac. The two cavity types are a $115 \mathrm{MHz}, \beta_{\mathrm{GEOM}}=0.15$ quarterwave resonant (QWR) cavity, and a $173 \mathrm{MHz}, \beta_{\mathrm{GEOM}}=$ 0.26 coaxial half-wave cavity. The useful velocity range of the two cavity types extends from 0.1 to $0.4 \mathrm{c}$. Both cavities are well-corrected for dipole and quadrupole asymmetries in the accelerating field. A cryomodule is being designed to incorporate a separate vacuum system for the cavity vacuum in order to provide a clean, lowparticulate environment for the superconducting cavities. This will enable a higher degree of surface cleanliness than has previously been the case for TEM-type, drifttube-loaded superconducting cavities. The status of prototype cavity and cryomodule construction are reported.
\end{abstract}

\section{INTRODUCTION}

The proposed U.S. Rare Isotope Accelerator (RIA) requires a driver linac formed of several hundred superconducting rf cavities of several different types to span a velocity range $0.02<\beta<0.9$. The driver will accelerate uranium ions to $400 \mathrm{MeV} /$ nucleon, and protons to energies of $900 \mathrm{MeV}$ [1].

Existing superconducting RF (SRF) technology is available for both the low-velocity and high-velocity sections of the driver linac. The low-velocity section is formed of cavities similar to the quarter-wave structures in existing heavy ion linacs[2], while the high-velocity section can employ $805 \mathrm{MHz}$ elliptical-cell cavity technology existing already developed at JLAB for the SNS project[3]. The cavities described in this paper fill an intermediate velocity range $0.1<\beta<0.4$. They are being designed to be processed with high-pressure water rinse cleaning techniques and to operate in the clean environment supported by separating the cavity and cryogenic vacuum systems.

\section{CAVITY PRODUCTION}

\section{Electrical and Mechanical Design}

Figure 1 shows the geometry of the two cavities, while Table 1 lists the calculated electromagnetic (EM) parameters. Pro/Engineer software was used to numerically model the mechanical properties of the structures, while the electromagnetic properties were modeled in 3D using CST Microwave Studio (Version 4).

The quarter-wave structure is useful over the velocity range $0.10<\beta<0.30$ while the half-wave covers $0.20<$ $\beta<0.40$. The drift-tube of the QWR structure is shaped
Table 1: EM Parameters (from Microwave Studio)

\begin{tabular}{|l|l|l|}
\hline & Quarter-Wave & Half-Wave \\
\hline Frequency [MHz] & 115 & 172.5 \\
\hline$\beta_{\text {GEOM }}$ & 0.15 & 0.26 \\
\hline Length [cm] & 25 & 30 \\
\hline $\mathbf{Q R}_{\mathbf{s}}$ & 42 & 58 \\
\hline At $1 \mathrm{MV} / \mathrm{m}$ accelerating field: \\
\hline $\mathbf{E}_{\text {peak }}[\mathbf{M V} / \mathbf{m}]$ & 3.2 & 2.9 \\
\hline $\mathbf{B}_{\text {peak }}[\mathbf{G}]$ & 57 & 78 \\
\hline rf energy [mJ] & 170 & 345 \\
\hline
\end{tabular}

to introduce electric dipole fields which largely cancel the beam steering caused by magnetic dipole fields intrinsic to the QWR geometry [4].

Design of the niobium shell and stainless-steel housing of both cavities followed analysis of the mechanical stresses both from helium pressure loads and also from mechanical tuning loads as shown in Figure 2.

\section{Fabrication}

The cavities have been designed for mass production.
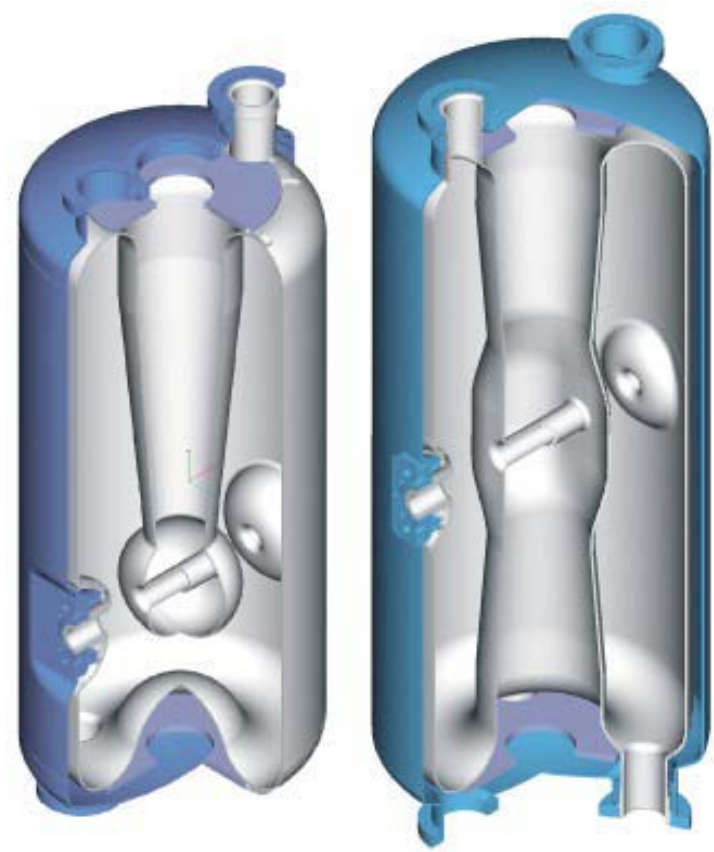

Figure 1: Cutaway views of the quarter-wave and halfwave cavities showing both the niobium cavity and the integral stainless-steel helium vessel. 
Figure 2: Numerically modeled displacement under a compressive load for tuning the QWR cavity.

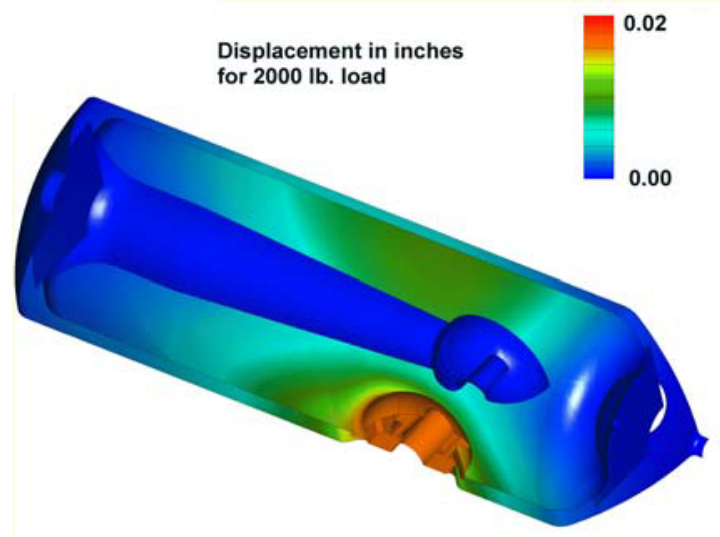

The blended corners and smooth transitions shown in Figure 1 are well suited to hydroforming and other sheet metal working processes, and also facilitate chemical processing and high-pressure water rinsing.

Dies and tooling were tested first on $3 \mathrm{~mm}$ aluminum sheet. Once parameters were established, the parts were formed of $3 \mathrm{~mm}$ niobium sheet with RRR $>250$. Figure 3 shows some of the sub-assemblies for the half wave cavity.

Formed parts were finish machined to fit, then joined with electron-beam welds. Figure 4 illustrates a complex weld on the quarter-wave center conductor involving multi-axis control of both gun and table with a varying beam current programmed to achieve optimum results.

These cavities are designed with integral stainless steel helium tanks which requiring joining niobium to stainless steel at the various coupling and beam ports. This was accomplished by brazing with pure copper, which requires neither plating or other surface treatment other than cleaning. Figure 5 shows two prototype braze assemblies. Copper wire is placed in a shoulder machined into the stainless steel and flows into the smooth annular space

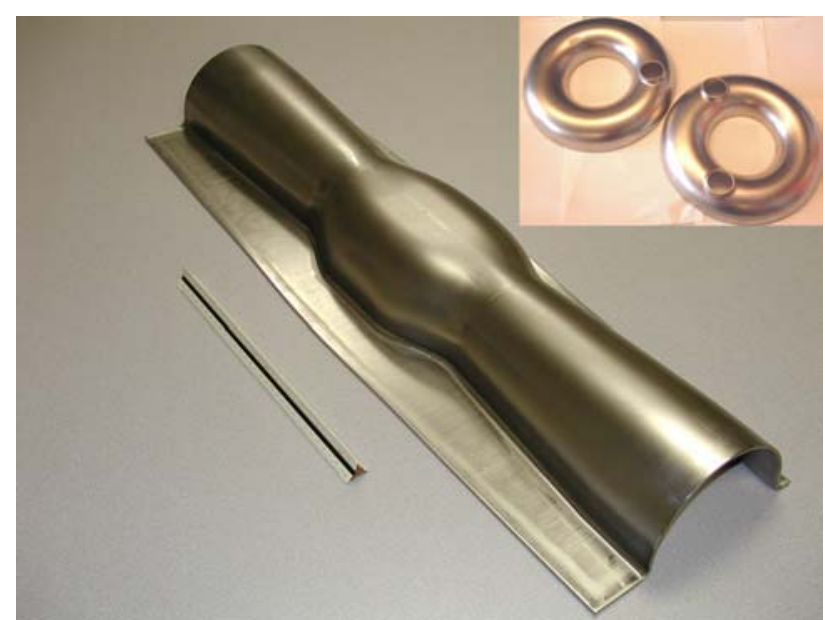

Figure 3: Half-wave resonator parts, as-formed niobium center conductor and (inset) toroidal end-cups with coupling ports.
Figure 4: Setup to electron-beam weld the QWR resonator center-conductor halves together in a single pump-down.

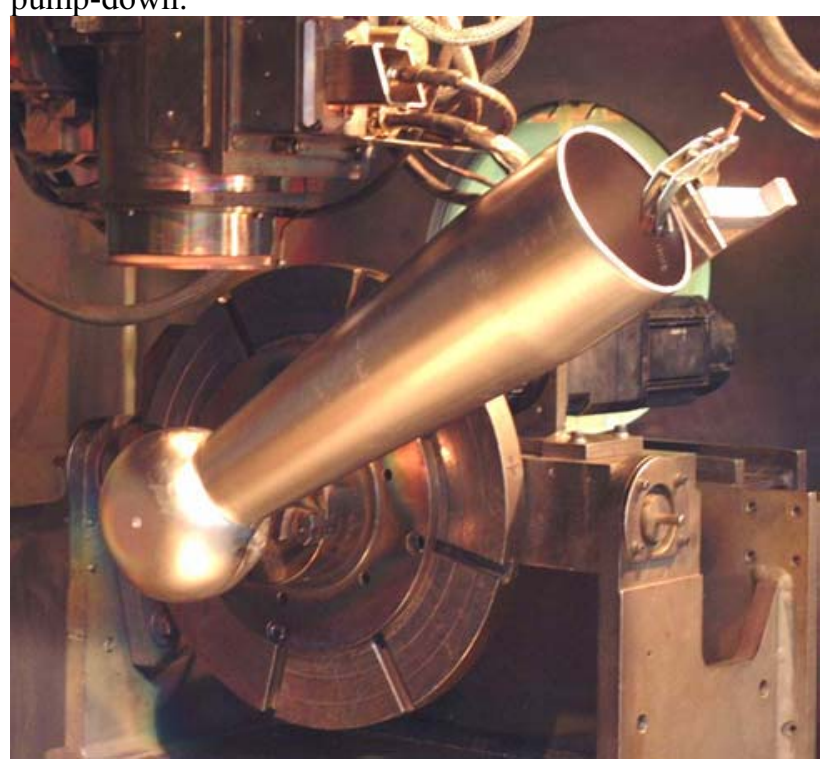

between stainless flange and niobium tube. The mating surfaces are completely wetted and the excess copper forms a fillet on the underside of the joint. Two beam port (30 $\mathrm{mm}$ ID) and three coupling port (51 mm ID) transitions of this type are currently in service and operating on a double spoke cavity at ANL [5].

\section{CRYOMODULE}

Low- $\beta$ drift-tube loaded TEM structures extend further vertically than along the beam axis, and can be efficiently housed in a rectangular cryostat geometry. Building on experience with the cryostats for the Positive Ion Injector at Argonne's ATLAS heavy ion linac, a rectangular cryomodule design has been developed [6] that is spaceefficient and consistent with the requirements for highperformance superconducting rf surfaces. Features include separation of the cavity and the cryogenic vacuum systems, and top-loading of the cavity-string subassembly which enables hermetic isolation of the cavity string along with assembly in a clean room.

Figure 5 shows three stages of module assembly, from clean-room subassembly to a completed module. The end walls of the vacuum vessel are chamfered in the middle, as shown in Figures 5 and 6 , to allow the roomtemperature, low-particulate beam-line vacuum valves which seal and isolated the cavity-string assembly, to insert through the cryomodule vacuum wall.

The cryomodule contains an internal magnetic shield as well as a liquid nitrogen cooled thermal shield. Both shields are installed by hanging sheets of copper and/or mu-metal, much like curtains, on the wall of the vacuum vessel, overlapping as required. Cost and installation effort should be reduced compared to more traditional construction methods. Multi-layer insulation (MLI) can be installed due to the independent cavity and cryogenic 
Figure 5: Cryomodule assembly sequence. (Top) cleanroom assembly of cavity string includes cavity and beamline vacuum and couplers. (Middle) Top flange assembly includes cryogenic systems. (Bottom) Completed cryomodule.

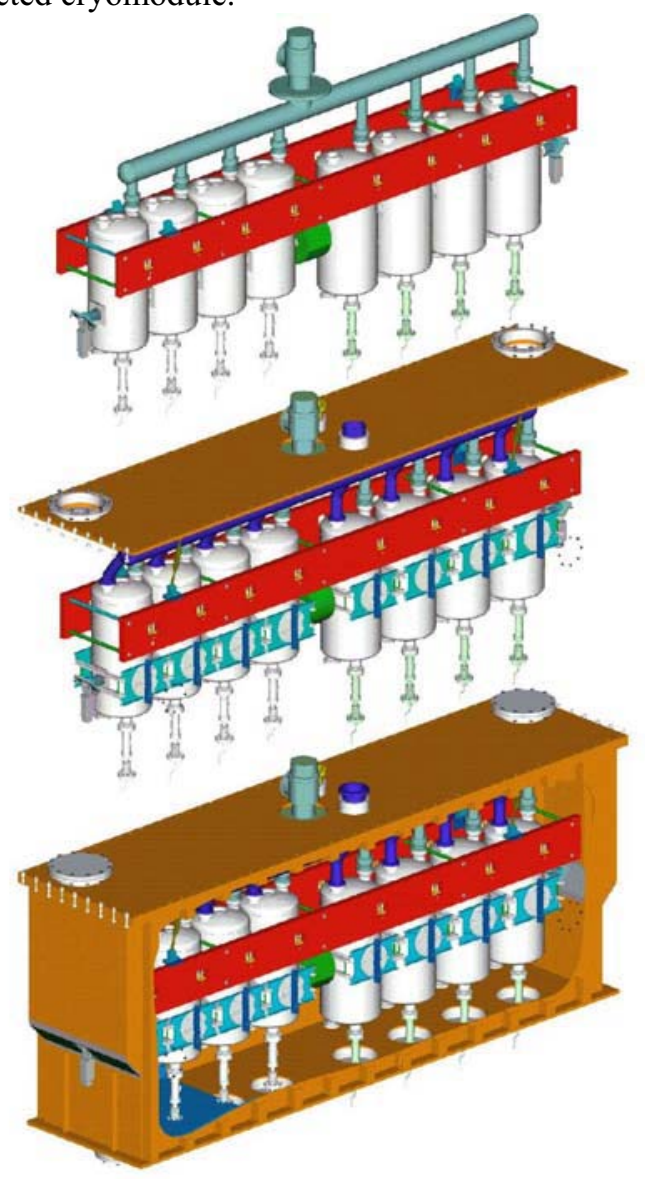

insulating vacuum spaces which ensure a clean cavity space.

\section{ACKNOWLEDGEMENTS}

We acknowledge the work of E. Peterson and his coworkers at Advanced Energy Systems, Inc. in designing tooling and forming niobium parts. T. Hejna of Sciaky, Inc developed procedures and performed electron beam welds for the work described in this paper. W. Toter of the ANL central shops developed the copper braze used to join stainless and niobium. Meyer Tool and Manufacturing Co. machined and fit many of the niobium parts.
Figure 6: Detail of cryomodule-cryomodule interface. The distance between SC cavity beam-port flange surfaces is $50.8 \mathrm{~cm}$. Some $20.6 \mathrm{~cm}$ of beamline between the $4 \mathrm{~cm}$, low-particulate beam-line valves is available for diagnostics, etc.

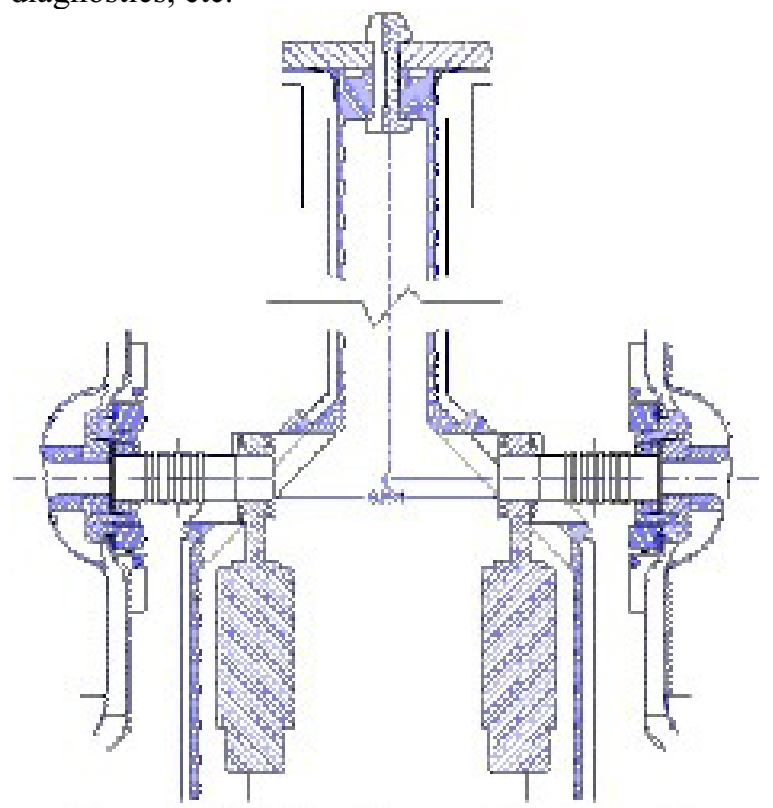

We also acknowledge many helpful discussions with numerous colleagues, including: J. Delayen, C. Rode, J. Preble, P. Kneisel and others at JLAB; D. Schrage, P. Kelly, and T. Tajima at LANL; and D. Proch and A. Mattheissen at DESY.

This work was supported by the U.S. Department of Energy under contract W-31-109-ENG-38.

\section{REFERENCES}

[1] C. W. Leemann, in Proc. 2000 Linac Conf., Monterey, California, August 21-25, 2000, SLAC VR-561, p.331 (2000).

[2] K.W. Shepard, Nucl. Intr. Meth. in Phys. Res. A 382, p125-131 (1996).

[3] G. Ciovatti, et al., in Proc. 10th Workshop on RF Superconductivity, Tsukuba, Japan, September 611, 2001, edited by S. Noguchi.

[4] P. N. Ostroumov and K.W. Shepard, Phys. Rev. ST AB 4, 110101 (2001).

[5] J.D. Fuerst et al, "Superconducting $345 \mathrm{MHz}$ TwoSpoke Cavity for RIA", in the proceedings of this conference.

[6] J.D. Fuerst and K.W. Shepard, "A Cryomodule for the RIA Driver Linac," ICEC19, Grenoble, July 2002. 